AIP Conference Proceedings

ISSN: 0094-243X (print); 1551-7616 (web)

Accepted December $10^{\text {th }} 2018$

\title{
FINITE ELEMENT ANALYSIS OF MELTING EFFECTS ON MHD STAGNATION-POINT NON-NEWTONIAN FLOW AND HEAT TRANSFER FROM A STRETCHING/SHRINKING SHEET Diksha Gupta ${ }^{1}$, Lokendra Kumar ${ }^{2, a)}$, O. Anwar Bég ${ }^{3}$ and Bani Singh 4
}

${ }^{1}$ Department of Mathematics, College of Science, University of Bahrain, 30238, Kingdom of Bahrain ${ }^{2}$ Department of Mathematics, Jaypee Institute of Information Technology, A-10, Sector-62, Noida-201309, Uttar Pradesh, India

${ }^{3}$ Fluid Mechanics, School of Computing, Science and Engineering, University of Salford, G77, Newton Building, M54WT, UK

${ }^{4}$ Department of Mathematics, Indian Institute of Technology, Roorkee-247667, India

${ }^{a)}$ Corresponding author: lokendma@gmail.com

\begin{abstract}
A numerical study is presented for boundary layer flow and heat transfer of micropolar (non-Newtonian) fluid from a stretching/shrinking sheet in the presence of melting and viscous heating. In this study the velocity of ambient fluid and stretching/shrinking velocity vary linearly with the distance from the stagnation-point. A uniform magnetic field is applied normal to the sheet and moves with the free stream as encountered in certain magnetic materials processing systems. Using similarity transformations, the governing partial differential equations are transformed into a system of coupled, nonlinear ordinary differential equations. A variational finite element code is implemented to solve the resulting dimensionless boundary value problem. The influence of magnetic body force $(\mathrm{M})$, stretching/shrinking $(\varepsilon)$ and melting (Me) parameters on velocity, microrotation, temperature, surface shear stress function (skin-friction) and local Nusselt number are elaborated in detail. Velocity is decreased with a rise in melting parameter, whereas far from the wall microrotation is reduced and furthermore temperatures are depressed. The flow is accelerated, micro-rotation (angular velocity of micro-elements) increased and temperature enhanced with increasing stretching rate $(\varepsilon>0)$ whereas the converse behaviour is observed with increasing shrinking rate $(\varepsilon<0)$. Increasing magnetic parameter is found to both increase temperatures and to accelerate the flow whereas it reduces microrotation near the wall and enhances it further from the wall. Special cases of the present model (with magnetic, dissipative and melting effects negated) are benchmarked with earlier results from the literature and found to be in excellent agreement. Excellent convergence and stability is achieved with the numerical method.
\end{abstract}


Keywords: Heat Transfer, Micropolar fluid, MHD, FEM.

\section{INTRODUCTION}

Heat transfer accompanied with melting effects arises in many diverse areas of chemical and mechanical engineering. These include cooling of micro-electronic circuits [1] and binary mixture synthesis [2]. Numerous experimental and theoretical studies of melting thermal convection flows have been communicated. Saxena et al. [3] studied melting of a cone in hot air, elaborating on the strong influence of melting and interface movement on the phase change fluid mechanics. Zhang and Bejan [4] examined theoretically and experimentally the effect of melting by natural convection. Cheng and Lin [5] studied numerically the influence of melting on unsteady convective flow from a vertical surface using the method of lines and a central finite difference technique. Bertrand et al. [6] studied computationally the combined natural convection and melting from an isothermal vertical wall in 2-dimensional enclosures. Takhar et al. [7] employed shooting quadrature and asymptotic methods to study melting effects on transient swirling convection flow from a disk. Bachok et al. [8] examined the influence of melting on the convective flow past a stretching/shrinking sheet.

The above studies were all confined to Newtonian flows. Melting convection flows in non-Newtonian fluids however also feature in many chemical technology processes. Many rheological models have been utilized for such simulations. Poulikakos and Spatz [9] studied the influence of non-Newtonian free convection past a melting surface. Kairi and Murthy [10] examined numerically the effect of melting and Soret on convection heat and mass transfer through a porous medium. Hayat et al. [11] used the homotopy semi-numerical technique to simulate melting effects on flow and heat transfer of viscoelastic fluid. Hayat et al. [12] also examined analytically the combined melting and viscous heating effects on stagnation-point third grade viscoelastic flow from a stretching surface, showing that increasing melting parameter serves to accelerate the flow and elevate momentum boundary layer thickness.

The rheological models described hitherto cannot simulate the complex microstructural characteristics of numerous polymeric materials. Eringen [13] developed the micropolar fluids theory. This theory has been deployed extensively to simulate a tremendous range of chemical engineering fluid dynamics problems. These include reactive diffusion flows [14], magnetohydrodynamic materials processing [15], stagnation flows [16], magnetic filtration flows [17], hydromagnetic generator flows [18], magneto-convective and transpiration-controlled plastic synthesis [19] and thin film electro-conductive polymer flows [20]. Further applications of micropolar fluid mechanics in polymeric materials flows are elaborated by Bég et al. [21] and furthermore in unsteady magnetized sheet stretching by Sharma et al. [22]. Although very sparse research has been reported on melting heat transfer in micropolar fluids, a recent effort in this regard has been made by Yacob et al. [23].

The shrinking sheet flows are of substantial concern in the manufacturing industries. The flow due to a shrinking sheet was first presented by Wang [24]. Later, Miklavčič and Wang [25] proved the existence of the solution for a shrinking sheet flow. Flow past a shrinking sheet was studied by Wang [26]. Micropolar fluid flow past a shrinking sheet was presented by Ishak et al. [27]. Mahapatra et al. [28] studied the effect of radiation on the flow and heat transfer over a shrinking sheet. Influence of convective boundary condition on the heat transfer of a viscous fluid flow over a stretching/shrinking sheet was investigated by Yao et al. [29].

The aim of the present paper is to study the effect of melting on the stagnation-point flow and heat transfer of an electrically conducting micropolar fluid past a stretching/shrinking sheet. It is assumed that the magnetic field moves with the free stream. This magnetic field scenario aids flow as opposed to the static magnetic field case which inhibits boundary layer flows [30]. Viscous heating is included. It is assumed that the melting of the sheet occurs at a steady rate i.e. transient effects are neglected. The conservation equations for mass, momentum, energy and angular momentum are transfered into a set of dimensionless non-linear ordinary differential equations which are solved under given boundary conditions with a variational finite element code. Under special conditions, the numerical results obtained for skin friction coefficient and the local Nusselt number are compared with those reported by Yacob et al. [23] and also Wang [24] demonstrating excellent correlation. Mesh independence of the numerical code is also demonstrated. The present work has applications in electro-conductive polymer thermal sheet processing [31, 32].

\section{MATHEMATICAL THERMO-FLUID DYNAMIC MODEL}

Consider the two-dimensional stagnation-point, steady flow of an incompressible micropolar fluid impinging normally on a stretching/shrinking sheet in the presence of magnetic field. This sheet is melting steadily into a constant property warm liquid of the same material. The $\mathrm{x}$-axis is taken in the direction of the sheet and $\mathrm{y}$-axis is taken normal 
to it. It is supposed that the stretching/shrinking velocity of the sheet is given by $u_{w}=c x$ and the external flow velocity is $u_{e}=a x$, where a positive value of $c$ represents stretching sheet while negative value implies a shrinking sheet and $a$ is a positive constant. Uniform magnetic field $B_{0}$ is applied in the positive y-direction normal to the sheet. It is assumed that magnetic field is moving with the free stream. For the present study the effect of the induced magnetic field can be neglected as compared to the imposed field. The temperature of the melting surface is $T_{m}$ while the free stream temperature is $T_{\infty}$ where $T_{\infty}$ exceeds $T_{m}$. Under the above assumptions the governing boundary layer equations may be presented as follows:

$$
\begin{aligned}
& \frac{\partial u}{\partial x}+\frac{\partial v}{\partial y}=0 \\
& u \frac{\partial u}{\partial x}+v \frac{\partial u}{\partial y}=u_{e} \frac{d u_{e}}{d x}+\frac{(\mu+S)}{\rho} \frac{\partial^{2} u}{\partial y^{2}}+\frac{S}{\rho} \frac{\partial N}{\partial y}+\frac{\sigma B_{0}^{2}}{\rho}\left(u_{e}-u\right) \\
& \left(u \frac{\partial N}{\partial x}+v \frac{\partial N}{\partial y}\right)=\frac{\gamma}{\rho j} \frac{\partial^{2} N}{\partial y^{2}}-\frac{S}{\rho j}\left(2 N+\frac{\partial u}{\partial y}\right) \\
& u \frac{\partial T}{\partial x}+v \frac{\partial T}{\partial y}=\frac{\kappa}{\rho c_{p}} \frac{\partial^{2} T}{\partial y^{2}}+\frac{(\mu+S)}{\rho c_{p}}\left(\frac{\partial u}{\partial y}\right)^{2}
\end{aligned}
$$

The relevant boundary conditions are given by:

$$
\begin{aligned}
& y=0: u=u_{w}(x)=c x, N=0, T=T_{m}, \kappa\left(\frac{\partial T}{\partial y}\right)_{y=0}=\rho\left[L+c_{s}\left(T_{m}-T_{0}\right)\right] v, \\
& y \rightarrow \infty: u=u_{e}(x)=a x, N=0, T=T_{\infty},
\end{aligned}
$$

where $L$ is the latent heat of the fluid and $c_{s}$ is the heat capacity of the solid surface. The last condition in eqn. (5) corresponds to the melting effect [23]. The velocity components $u$ and $v$ can be expressed in the form of the stream function $\phi$ such that

$$
u=\frac{\partial \phi}{\partial y}, v=-\frac{\partial \phi}{\partial x} \text {. }
$$

Clearly, the continuity equation (1) is satisfied automatically. To normalize the boundary value problem defined by eqns (1) to (6), we select and implement a suitable group of coordinate and variable transformations, where all terms are defined in the notation:

$\xi=\sqrt{\frac{a}{v}} x, \eta=\sqrt{\frac{a}{v}} y, \phi=\sqrt{v a} x f, N=\sqrt{\frac{a}{v}}$ ax $g, \theta=\frac{T-T_{m}}{T_{\infty}-T_{m}}$.

This leads to the following group of coupled momentum, angular momentum and energy ordinary differential equations:

$$
\begin{aligned}
& (1+K) f^{\prime \prime \prime}+f f^{\prime \prime}-\left(f^{\prime}\right)^{2}+K g^{\prime}+M\left(1-f^{\prime}\right)+1=0, \\
& A g^{\prime \prime}+C\left(f g^{\prime}-f^{\prime} g\right)-K\left(2 g+f^{\prime \prime}\right)=0, \\
& \theta^{\prime \prime}+\operatorname{Pr} f \theta^{\prime}+(1+K) \operatorname{Pr} \operatorname{Ec}\left(f^{\prime \prime}\right)^{2}=0,
\end{aligned}
$$

The corresponding non-dimensional boundary conditions at the wall and in the free stream become:

$$
\begin{aligned}
& f^{\prime}(0)=\varepsilon, g(0)=0, \operatorname{Pr} f(0)+M e \theta^{\prime}(0)=0, \theta(0)=0, \\
& f^{\prime}(\infty)=1, g(\infty)=0, \theta(\infty)=1,
\end{aligned}
$$


where dash depicts the differentiation with respect to $\eta$ only, $K=S / \mu$ is the micropolar coupling constant parameter, $M=\sigma_{0} B_{o}^{2} / \rho a$ is the magnetic body force parameter, $A=\gamma a \rho / \mu^{2}$ is the micropolar spin gradient viscosity parameter, $C=\rho j a / \mu$, is the microinertia density parameter, $E c=u_{e}^{2} / c_{p}\left(T_{\infty}-T_{m}\right)$ is the Eckert number, $\operatorname{Pr}=\mu c_{p} / \kappa$ is the Prandtl number, $\varepsilon=c / a ; \varepsilon>0$ [for stretching and $\varepsilon<0$ for shrinking], $M e=c_{f}\left(T_{\infty}-T_{m}\right) /\left(L+c_{s}\left(T_{m}-T_{0}\right)\right)$ is the melting parameter which is a combination of the Stefan numbers $c_{f}\left(T_{\infty}-T_{m}\right) / L$ for liquid phase and $c_{s}\left(T_{m}-T_{0}\right) / L$ solid phase. The skin-friction coefficient and local Nusselt number are physical important parameters for materials processing flows, and are defined, respectively by:

$$
C_{f}=\frac{\tau_{w}}{\rho u_{e}^{2}}, N u_{x}=\frac{q_{w} x}{\kappa\left(T_{\infty}-T_{m}\right)}
$$

where $\tau_{w}$ and $q_{w}$ are the wall shear stress and heat flux. Using the similarity transformations (10), along with the following definitions:

$$
\tau_{w}=-\left[(\mu+S) \frac{\partial u}{\partial y}+S N\right]_{y=0} \text { and } q_{w}(x)=-\kappa\left(\frac{\partial T}{\partial y}\right)_{y=0}
$$

It follows that:

$$
\begin{aligned}
& C_{f}\left(\operatorname{Re}_{x}\right)^{1 / 2}=(1+K) f^{\prime \prime}(0), \\
& \frac{N u_{x}}{\left(\operatorname{Re}_{x}\right)^{1 / 2}}=-\theta^{\prime}(0),
\end{aligned}
$$

where $\operatorname{Re}_{x}=a x^{2} / v$ is the local Reynolds number.

\section{VARIATIONAL FINITE ELEMENT COMPUTATIONAL SOLUTIONS}

The collective set of differential eqns. (9)-(11) is strongly non-linear and therefore, the finite element method has been employed for a numerical solution. Comprehensive details of this very popular and efficient technique are provided in Reddy [33]. The version employed herein is a variational formulation which has met with considerable success in recent years in simulating many complex fluid flows. These include thermal radiation-convection micropolar flow [34], mixed convection micropolar flow [35], magneto-hemodynamic non-Newtonian flow [36], nanofluid mechanics [36-38] and free surface wave hydrodynamics [39].

In order to apply finite element method first we assume

$f^{\prime}=h$.

Thus the set of equations (9)-(11) reduce to:

$$
\begin{aligned}
& (1+K) h^{\prime \prime}+f h^{\prime}-h^{2}+K g^{\prime}+M(1-h)+1=0, \\
& A g^{\prime \prime}+C\left(f g^{\prime}-h g\right)-K\left(2 g+h^{\prime}\right)=0, \\
& \theta^{\prime \prime}+\operatorname{Pr} f \theta^{\prime}+(1+K) \operatorname{Pr} E c\left(h^{\prime}\right)^{2}=0,
\end{aligned}
$$

The corresponding boundary conditions become:

$$
\begin{aligned}
& h(0)=\varepsilon, g(0)=0, \operatorname{Pr} f(0)+M e \theta^{\prime}(0)=0, \theta(0)=0, \\
& h(\infty)=1, g(\infty)=0, \theta(\infty)=1 .
\end{aligned}
$$

The system of equations obtained after the assembly of the elements is non-linear and a robust iterative scheme is employed for a solution. In order to check the convergence of the results the computations have been performed for 20, 40, 60, 80, 100, 120, 140, 160, 180, 200 elements and are presented in Table 1. It is observed that by increasing the number of elements beyond 160 there is no significant change in the value of $f, h, g$ and $\theta$, therefore the final results for 160 elements suffice. Mesh independence of the numerical code is therefore achieved. 
Table 2 and $\mathbf{3}$ show the comparison of local skin friction and local Nusselt number values with the present FEM results and the previously published work of Yacob et al. [23] for non-magnetic, non-dissipative micropolar melting heat transfer and also Wang [24] for non-magnetic, non-dissipative, Newtonian flow without melting. It is clear from the tables that the numerical results computed are in excellent agreement with the data available in the literature.

Table 1: Convergence of results with the variation of number of elements $n$

\begin{tabular}{ccccc}
\multicolumn{5}{c}{$(K=1, M=3, A=1, C=1, \operatorname{Pr}=0.733, E c=0.02, \varepsilon=-0.5, M e=1)$} \\
\hline$n$ & $f(1.6)$ & $h(1.6)$ & $g(1.6)$ & $\theta(1.6)$ \\
\hline 20 & 0.24752 & 0.88268 & -0.09302 & 0.54839 \\
40 & 0.24132 & 0.87860 & -0.09197 & 0.54480 \\
60 & 0.23956 & 0.87782 & -0.09181 & 0.54396 \\
80 & 0.23851 & 0.87741 & -0.09174 & 0.54349 \\
100 & 0.23832 & 0.87734 & -0.09172 & 0.54340 \\
120 & 0.23820 & 0.87730 & -0.09172 & 0.54335 \\
140 & 0.23812 & 0.87727 & -0.09171 & 0.54331 \\
160 & 0.23806 & 0.87725 & -0.09171 & 0.54329 \\
180 & 0.23802 & 0.87724 & -0.09171 & 0.54327 \\
200 & 0.23799 & 0.87723 & -0.09171 & 0.54326 \\
\hline
\end{tabular}

Table 2: Comparison of $f^{\prime \prime}(0)$ for different values of $\varepsilon(K=0, M=0, E c=0, M e=0)$

\begin{tabular}{cccc}
\hline$\varepsilon$ & Wang [26] & Yacob et al. [23] & Present results \\
\hline 0 & 1.232588 & 1.232588 & 1.232784 \\
0.50 & 0.71330 & 0.713295 & 0.713148 \\
2.00 & -1.88731 & -1.887307 & -1.884940 \\
5.00 & -10.26475 & -10.264749 & -10.231600 \\
-0.25 & 1.40224 & 1.402241 & 1.402736 \\
-0.50 & 1.49567 & 1.495670 & 1.496505 \\
-0.75 & 1.48930 & 1.489298 & 1.490471 \\
-1.00 & 1.32882 & 1.328817 & 1.330265 \\
-1.15 & 1.08223 & 1.082231 & 1.083805 \\
\hline
\end{tabular}

Table 3: Comparison of $-\theta^{\prime}(0)$ for different values of $\varepsilon(K=0, M=0, E c=0, M e=0)$

\begin{tabular}{ccc}
\hline$\varepsilon$ & Yacob et al. $[23]$ & Present results \\
\hline 0 & -0.570465 & -0.570609 \\
0.50 & -0.692964 & -0.692454 \\
2.00 & -0.979271 & -0.980752 \\
5.00 & -1.396355 & -1.400973 \\
\hline
\end{tabular}




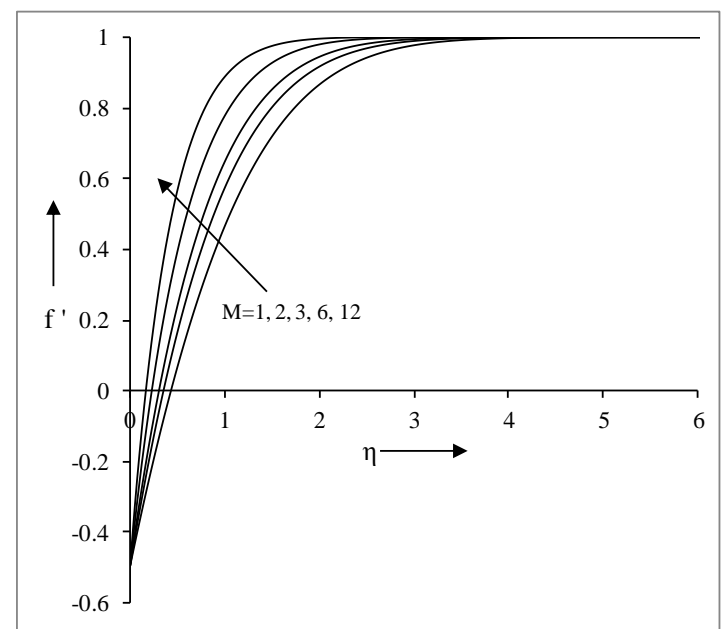

FIGURE 1. Velocity distribution for different $M(\varepsilon=-0.5, M e=1)$
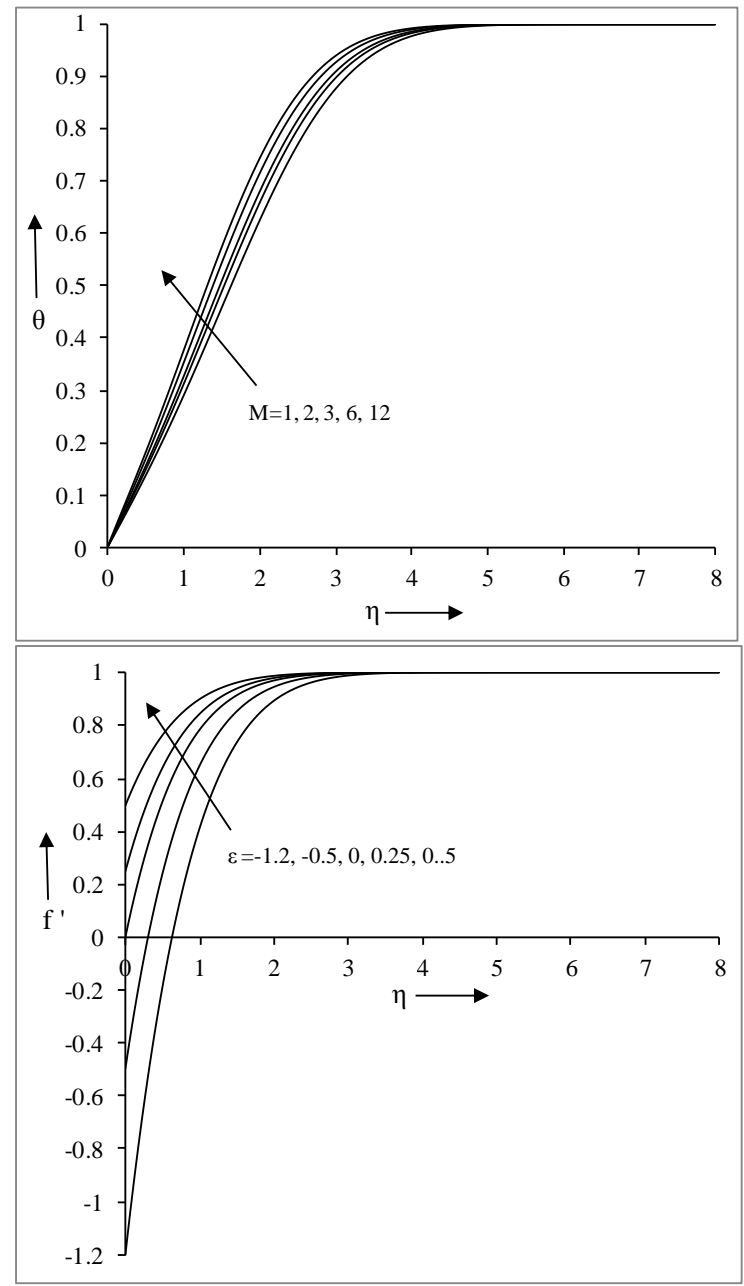

FIGURE4. Velocity distribution for different

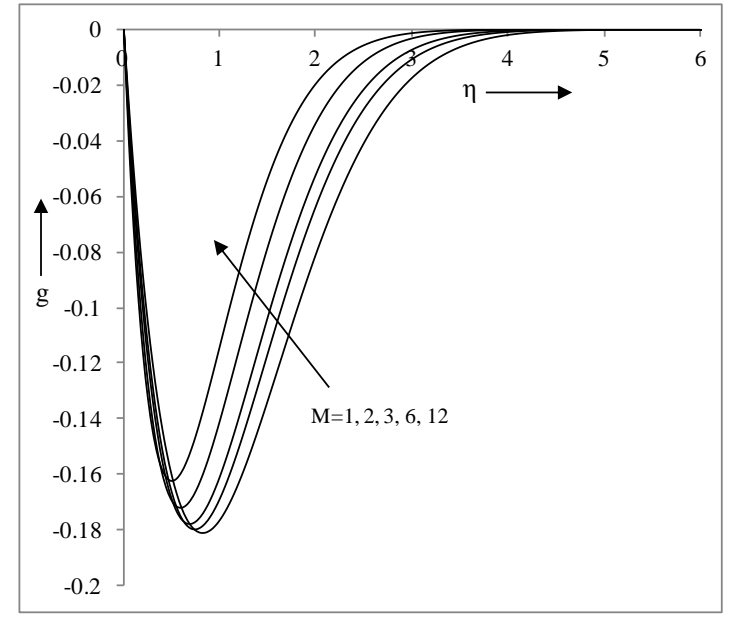

FIGURE 2. Microrotation distribution for different $M(\varepsilon=-0.5, M e=1)$

FIGURE 3. Temperature distribution for different 


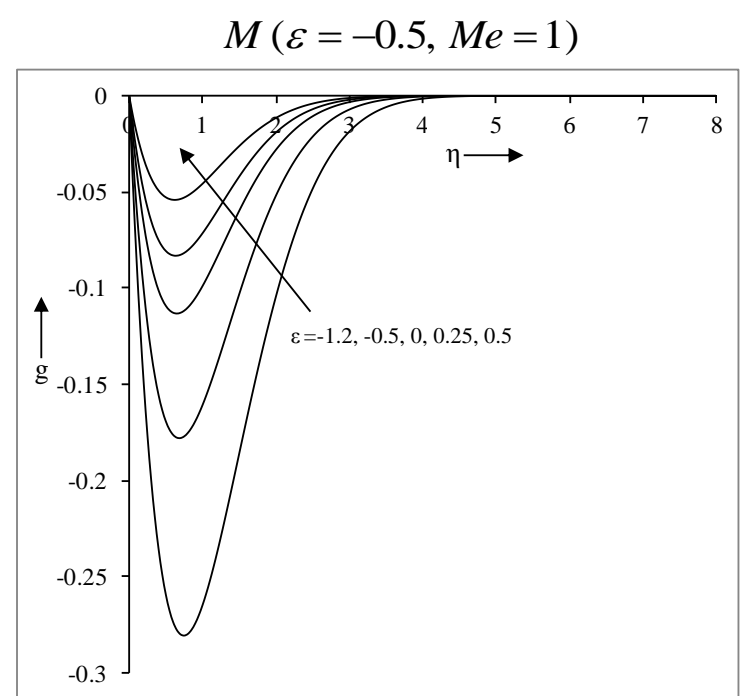

FIGURE 5. Microrotation distribution for different different $\quad \varepsilon(M=3, M e=1)$

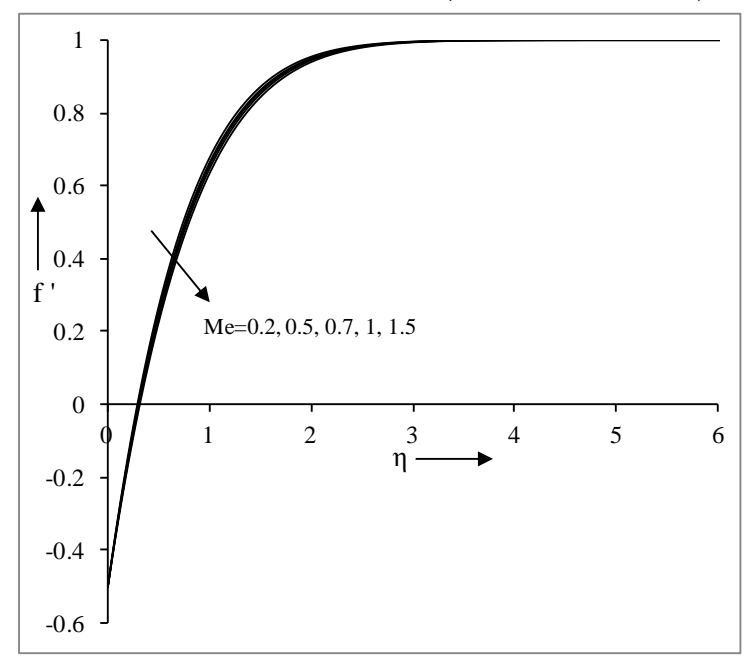

FIGURE 7. Velocity distribution for different $\operatorname{Me}(M=3, \varepsilon=-0.5)$
$\varepsilon(M=3, M e=1)$

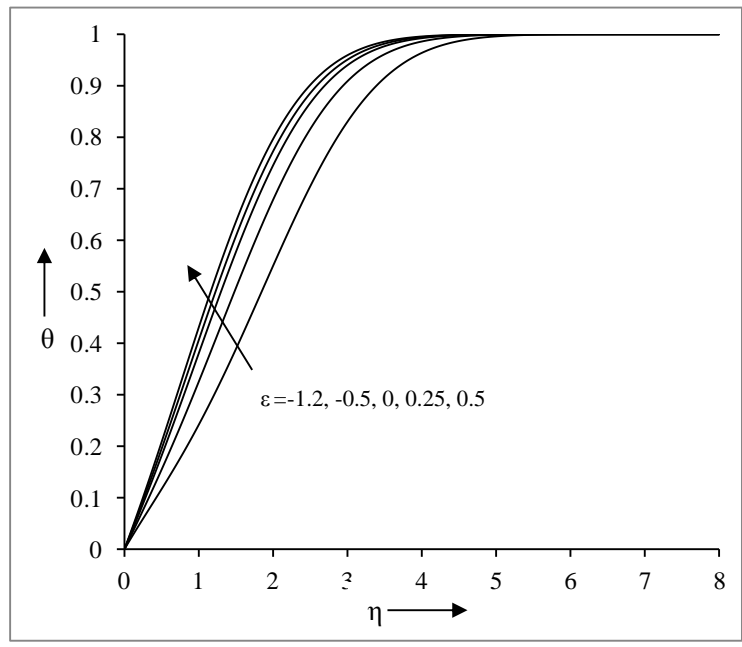

FIGURE6. Temperature distribution for $\varepsilon(M=3, M e=1)$

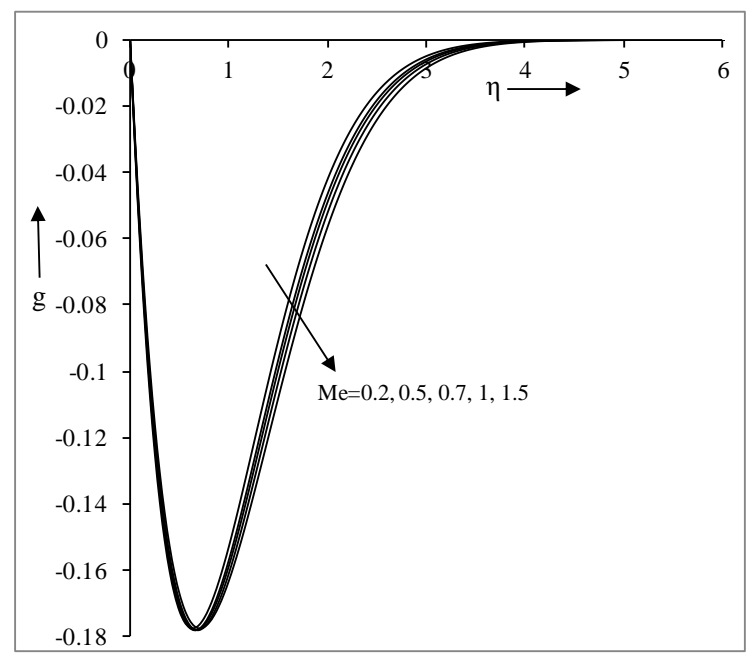

FIGURE 8. Microrotation distribution for different $M e(M=3, \varepsilon=-0.5)$

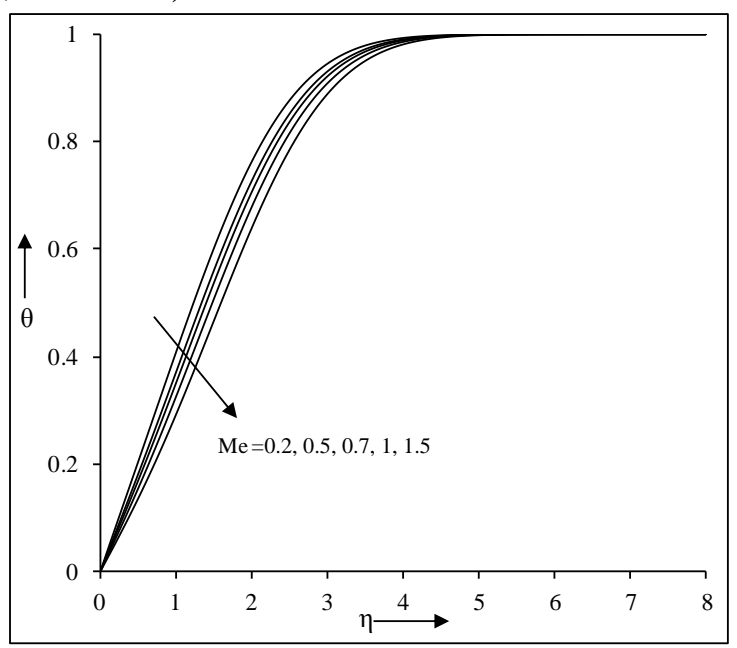

FIGURE 9. Temperature distribution for different 
$\operatorname{Me}(M=3, \varepsilon=-0.5)$

\section{RESULTS AND DISCUSSION}

In order to have a physical point of view of the problem, rigorous numerical calculations have been performed for velocity, microrotation and temperature function distributions across the boundary layer for different values of the thermophysical parameters i.e. magnetic parameter $M$, stretching/shrinking parameter $\varepsilon$ and melting parameter $M e$. These results are shown graphically in Figs. 1-9. Streamlines are shown in Figs. 10-11. Tables 4-5 show the skin-friction coefficient and local Nusselt number for various values of the parameters. In the present study, coupling constant parameter, $\operatorname{Pr}$ and $E c$ are kept fixed as $1,0.733$ and 0.02 , whereas values of spin gradient viscosity and microinertia density parameters are fixed at unity. This approach follows the established simulations of previous works in the literature including Pal et al. [18] and Yacob et al. [24].

The effect of magnetic parameter $M$ on the velocity evolution is examined in Fig. 1. Velocity increases and the momentum boundary layer thickness also increases with increase in the magnetic parameter, $M$. The magnetic field is mobile and tends to drag the fluid with it. The body force term, $M\left(1-f^{\prime}\right)$ shows that for $f^{\prime}<1$, the contribution of magnetism is to assist the momentum development (the Lorentzian force is positive for $\left(f^{\prime}<1\right)$. This effectively accelerates the flow. Larger magnetic field serves to decrease the momentum boundary layer thickness and enhances velocity gradient. The shear stress at the wall will also be elevated as the fluid is accelerated and this effect is amplified as the fluid transfers momentum to adjacent layers through the action of viscosity, with progressive distance into the boundary layer transverse to the wall. In materials processing operations, the shrinking sheet ( $\varepsilon=-0.5$ in fig. 1 ) flow is therefore accelerated with stronger magnetic field strength. Fig. 1 also corresponds to strong melting effects $(M e=1.0)$. Fig. 2 depicts the microrotation profiles for different values of magnetic parameter, $M$. As $M$ rises, microrotation is strongly accentuated in the boundary layer. The acceleration in the momentum field indirectly influences the micro-rotation field via the coupling term, $K g^{\prime}$, in eqn. (9) and furthermore via the coupling terms in eqn (10), $+C\left(f g^{\prime}-f^{\prime} g\right)$ and $-K\left(f^{\prime \prime}\right)$. The momentum boost will aid the spin of microelements as does distance from the wall. Rotary motions are therefore enhanced strongly and angular momentum boundary layer thickness is decreased with increasing magnetic body force effect. For the case $M=1$, the magnetic body force and viscous hydrodynamic force in the regime are of equal magnitude and the angular velocity (microrotation) is found to be minimized. Angular momentum boundary layer thickness will therefore be a maximum for this weakest magnetic field case. Fig. 3 illustrates the strong enhancement in temperature $(\theta)$ again for the shrinking sheet case, with increasing magnetic field parameter, $M$. Similar observation has been made by Das [40]. In all the computations, $\operatorname{Pr}$ has been prescribed a value of 0.733 , representative of certain low molecular weight polymers. The increase in temperature with magnetic field however will also increase heat transfer rate from the wall to the fluid. This will decrease thermal boundary layer thickness.

Figs. 4-6 illustrate the effect of stretching/shrinking parameter $(\varepsilon)$ on the boundary layer characteristics. In fig 4 , the variations of velocity within the boundary layer for different values of stretching/shrinking parameter $(\varepsilon)$ are presented. Positive values of $\varepsilon$ imply stretching of the sheet and negative values correspond to shrinking of the sheet. The case of $\varepsilon=0$ implies a stationary wall without magnetic field, melting and non-Newtonian effects and this classical scenario is known as Hiemenz flow (stagnation flow on a solid surface). Velocity is considerably elevated with increase in the stretching rate, since this causes decrease in the momentum boundary layer thickness, as a result of which shear stresses are enhanced and the flow is accelerated. The converse behaviour is observed with increasing shrinking rate. Inspection of Fig. 5 shows that microrotation (angular velocity, $g$ ) increases with increase in stretching rate $(\varepsilon>0)$ whereas it is significantly suppressed with increase in shrinking rate. Negative value of microrotation indicates the reverse rotation of microelements. Although negative values are sustained throughout the boundary layer irrespective of sheet stretching or shrinkage, the intensity of reverse spin is inhibited with stretching but accentuated with shrinking. Micro-element rotary motions are therefore benefited with greater stretching of the sheet but opposed with greater shrinkage. The profile for the solid wall case ( $\varepsilon=0$ ) naturally falls between the stretching and shrinking cases. Fig. 6 reveals that micropolar fluid temperature increases as stretching rate increases whereas it is depressed with increase in the shrinking rate. This can be explained by the fact that the thermal boundary layer becomes thinner as stretching rate increases with a concomitant rise in fluid temperatures. On the other hand, thermal boundary layer 
thickness is enhanced with increasing shrinking rate. The extension or contraction of the sheet effectively therefore exerts a major effect on flow and heat transfer properties in the region close to the sheet and is a principal control mechanism in practical operations.

Figs. 7-9 depict the influence of the melting parameter on the velocity, angular velocity and temperature fields, for the shrinking sheet case $(\varepsilon<0)$ with strong magnetic field $(M=3)$. In fig. 7 it is apparent that the velocity reduces with rise in melting parameter. This trend has also been identified by Bachok et al. [8]. Enhanced melting forces a deceleration in the flow as more material is converted into fluid. This thickens the momentum boundary layer, enhances wall shear stress and serves to weakly damp the flow. A same result has also been given by Cheng and Lin [5] and Takhar et al. [7]. In all the graphs, the boundary conditions (22) are satisfied asymptotically in the free stream which confirms the convergence of the computations. The melting parameter does not feature in the conservation eqns. (18)-(20), and only arises in the wall boundary condition (21) i.e. $\operatorname{Pr} f(0)+M e \theta^{\prime}(0)=0$. Inspite of this the parameter, $M e$, still exerts an influence far from the wall. This is also observed in the micro-rotation and temperature fields. It is observed from Fig. 8 that in the vicinity of the sheet effect of melting parameter on the microrotation is almost negligible whereas far from the sheet microrotation decreases strongly with increase in melting parameter. The negative values as elaborated earlier correspond to a reversal in micro-element spin. The effect of $M e$ on the temperature distribution (Fig. 9) is to decrease the temperature of the fluid. Similar observations were reported by Hayat et al. [11].

In the presence of melting the streamlines for shrinking and stretching sheet scenarios are shown in Figs. 10 (a, b), whereas in absence of melting they are illustrated in Figs. $11(\mathbf{a}, \mathbf{b})$. Due to reflective symmetry only a half portion of the flow is visualized in the figures. In Figs. $10(\mathbf{a}, \mathbf{b})$ the tendency of more intense flow towards the streamwise direction further from the shrinking sheet with melting is observed. For the stretching sheet (fig. 10b) the flow converges to a narrower band near the sheet. From Fig. 11 (a) it appears that flow reversal takes place near the shrinking sheet. There is a discontinuity in the flow near the sheet. However with stretching the streamlines all converge consistently towards the downstream direction. These patterns are consistent with the findings of Mahapatra et al. [28].

Table 4 presents the influence of magnetic, stretching/shrinking and melting parameters on the skin friction $\left\{f^{\prime \prime}(0)\right\}$. The skin friction decreases with rise in melting and stretching rate, whereas it increases with rise in $M$ and shrinking rate. Hence skin friction can be minimized effectively by judicious selection of the melting and stretching parameters. From Table 5 it may be noted that the rate of heat transfer increases numerically as magnetic parameter and stretching rate increase, while it decreases with rise in melting parameter and shrinking rate. For smaller values of melting parameter, heat transfer rate is higher. This observation matches with the results obtained by Yacob et al. [23]. Negative values of the heat transfer rate indicate that the heat is transferred from the fluid to the solid surface (sheet). 


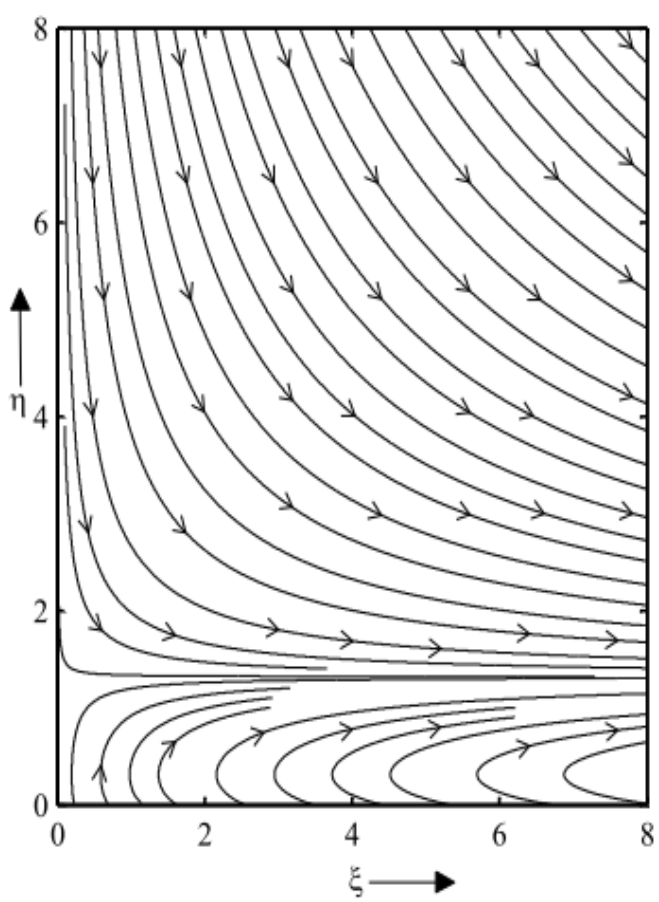

(a)

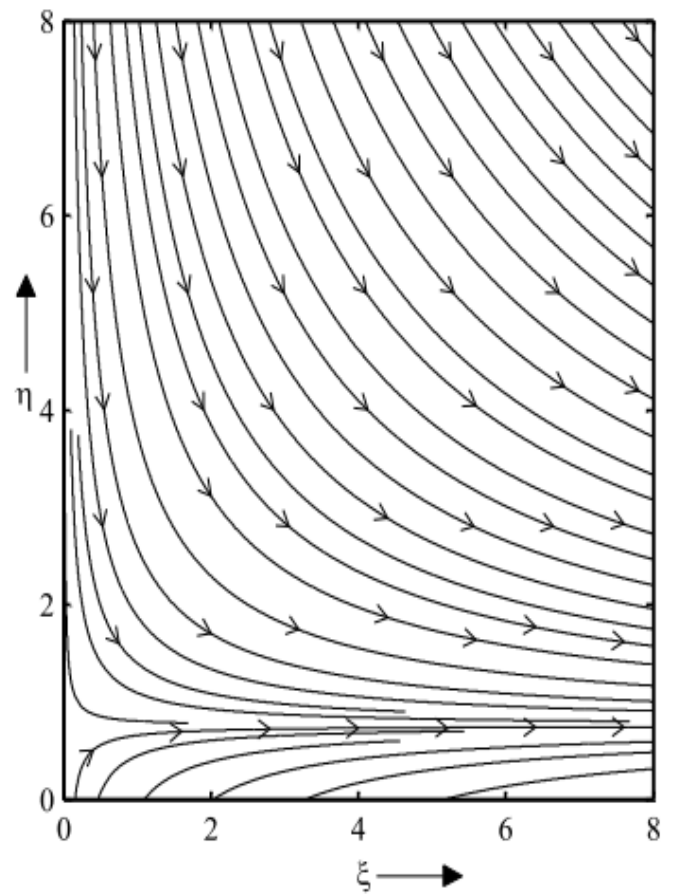

(b)

Fig. 10. Streamlines for $\mathrm{Me}=1, \mathrm{M}=3$ and (a) $\varepsilon=-0.5$ (b) $\varepsilon=0.5$

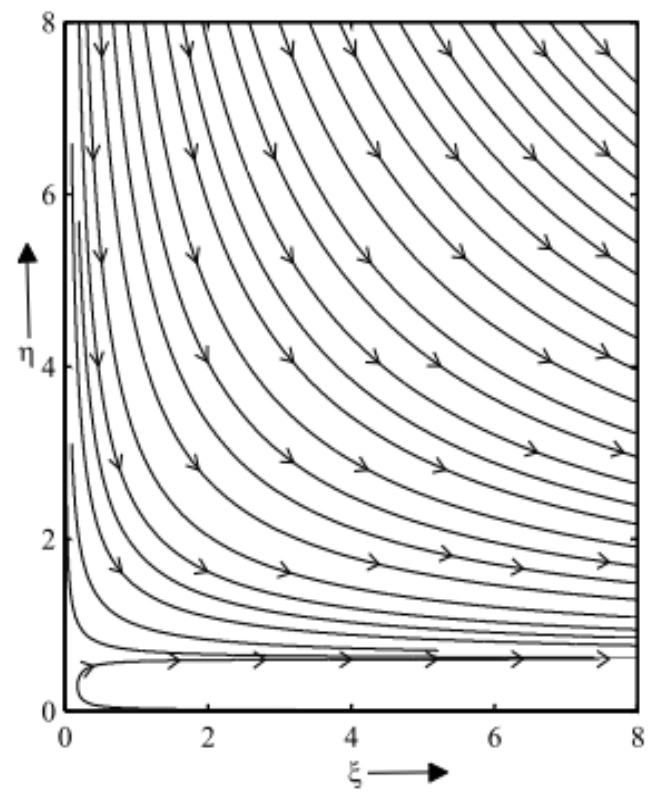

(a)

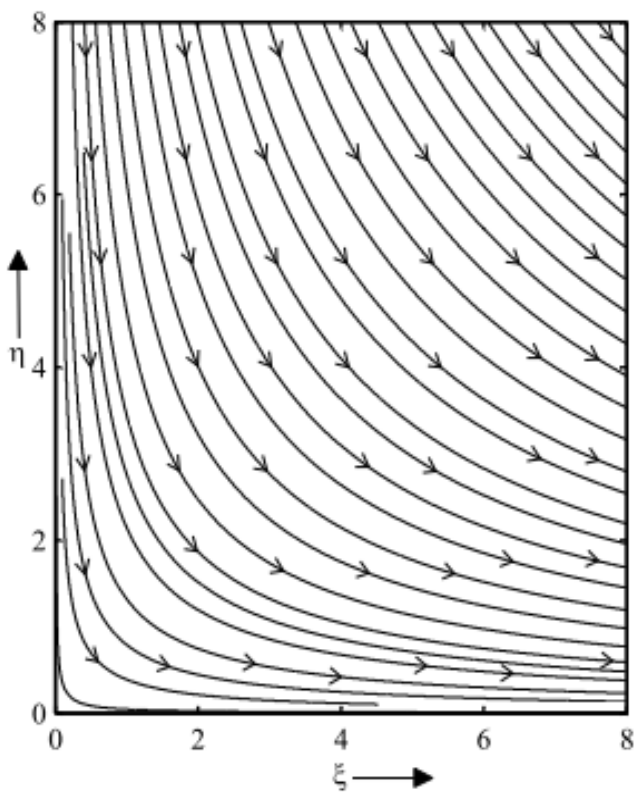

(b)

Fig. 11. Streamlines for $\mathrm{Me}=0, \mathrm{M}=3$ and (a) $\varepsilon=-0.5$ (b) $\varepsilon=0.5$

Table 4: The skin friction coefficient $f^{\prime \prime}(0)$ for different values of $M, \quad \varepsilon$ and $M e$ $(K=1, A=1, C=1, \operatorname{Pr}=0.733, E c=0.02)$ 


\begin{tabular}{cccccc}
\hline \multicolumn{2}{c}{$\varepsilon=-0.5, M e=1$} & \multicolumn{2}{c}{$M=3, M e=1$} & \multicolumn{2}{c}{$M=3, \varepsilon=-0.5$} \\
\hline$M$ & $f^{\prime \prime}(0)$ & $\varepsilon$ & $f^{\prime \prime}(0)$ & $M e$ & $f^{\prime \prime}(0)$ \\
\hline 1 & 1.293535 & -1.20 & 2.527196 & 0.2 & 2.015478 \\
2 & 1.623619 & -0.50 & 1.901793 & 0.5 & 1.963669 \\
3 & 1.901793 & 0.00 & 1.344154 & 0.7 & 1.936108 \\
6 & 2.572453 & 0.25 & 1.034979 & 1.0 & 1.901793 \\
12 & 3.568898 & 0.50 & 0.707193 & 1.5 & 1.857108 \\
\hline
\end{tabular}

Table 5: The local Nusselt number $-\theta^{\prime}(0)$ for different values of $M, \quad \varepsilon$ and $M e$ $(K=1, A=1, C=1, \operatorname{Pr}=0.733, E c=0.02)$

\begin{tabular}{cccccc}
\hline \multicolumn{2}{c}{$\varepsilon=-0.5, M e=1$} & \multicolumn{2}{c}{$M=3, M e=1$} & \multicolumn{2}{c}{$M=3, \varepsilon=-0.5$} \\
\hline$M$ & $-\theta^{\prime}(0)$ & $\varepsilon$ & $-\theta^{\prime}(0)$ & $M e$ & $-\theta^{\prime}(0)$ \\
\hline 1 & -0.262633 & -1.20 & -0.247543 & 0.2 & -0.417924 \\
2 & -0.285805 & -0.50 & -0.302602 & 0.5 & -0.363580 \\
3 & -0.302602 & 0.00 & -0.343835 & 0.7 & -0.335852 \\
6 & -0.336089 & 0.25 & -0.365199 & 1.0 & -0.302602 \\
12 & -0.374561 & 0.50 & -0.387205 & 1.5 & -0.261611 \\
\hline
\end{tabular}

\section{CONCLUSIONS}

In the present work we have addressed the influence of melting heat transfer on the MHD stagnation-point micropolar fluid flow over a stretching/shrinking sheet with viscous dissipation. It is found that the drag can be reduced effectively with melting parameter and stretching rate. It has also been observed that the rate of heat transfer can be increased with the magnetic parameter and stretching rate. The results obtained in the present investigation may be helpful in the selection of a micropolar fluid with suitable combinations of various parameters for achieving drag reduction and heat transfer rate augmentation in materials processing systems. The study reported here has ignored slip effects [40] at the sheet. These may be considered in the future.

\section{REFERENCES}

[1] R. Chebi, P.A. Rice and J.A. Schwarz, Chemical Engineering Communications 69, 1-12 (1988).

[2] H.J. Kim, S.H. Youn and C.S. Shin, Chemical Engineering J.146, 449-455 (2009).

[3] S. Saxena, M.K. Sarkar andS. Subrahmaniyam, Chemical Engineering Communications 24, 369-376 (1983).

[4] Z. Zhang and A. Bejan, Int. J. Heat Mass Transfer 32, 2447-2457 (1989).

[5] W.T. Cheng and C.H. Lin, Int. J. Eng. Sci. 44, 1023-1036 (2006).

[6] O. Bertrand, B. Binet, H. Combeau, S. Couturier, Y. Delannoy, D. Gobin, L. Marcel and G. Vieira, Int. J. Thermal Sciences 38, 5-26 (1999).

[7] H.S. Takhar, M. Kumari and G. Nath, Communications in Numerical Methods in Engineering 16, 485-496 (2000).

[8] N. Bachok, A. Ishak and Pop, I., Phy. Lett. A 374, 4075-4079 (2010).

[9] D. Poulikakos and T.L. Spatz, Int. Commun. Heat Mass Transfer 15, 593-603 (1988).

[10] R.R. Kairi and P.V.S.N. Murthy, ASME J. Heat Transfer 134, 042601 (2012).

[11] T. Hayat, M. Mustafa, S.A. Shehzad and S. Obaidat, Int. J. Numer. Meth. Fluids 68, 233-243 (2012).

[12] T. Hayat, Z. Iqbal, M. Mustafa and A.A. Hendi, Thermal Science 17, 865-875 (2013).

[13] A.C. Eringen, J. Math. Mech. 16, 1-18 (1966). 
[14] M.M. Rahman and M. Al-Lawatia, Canadian J. Chemical Engineering 88, 23-32 (2010).

[15] N.A. Yacob and A. Ishak, Proc IChemE-Chem. Eng. Res. Des. 89, 2291-2297 (2011).

[16] N.A. Yacob and A. Ishak, Canadian J. Chemical Engineering 90, 621-626 (2012).

[17] J. Zueco, O. Anwar Bég and H.S. Takhar, Computational Materials Science 46, 1028-1037 (2009).

[18] D. Pal, B. Talukdar, I.S. Shivakumara and K. Vajravelu, Chemical Engineering Communications 199, 943-965 (2012).

[19] M.A. Seddeek, S.N. Odda, M.Y. Akl and M.S. Abdelmeguid, Computational Materials Science 45, 423-428 (2009).

[20] O.A. Bég, J. Zueco and T.B. Chang, Chemical Engineering Communications 198, 312-331(2011).

[21] O.A. Bég, V.R. Prasad, B. Vasu, N.B. Reddy, Q. Li and R. Bhargava, Int. J. Heat Mass Transfer 54, 9-18 (2011).

[22] R. Sharma, R. Bhargava and I.V. Singh, Applied Mathematics and Computation 217, 308-321 (2012).

[23] N.A. Yacob, A. Ishak and I. Pop, Comput. Fluids 47, 16-21(2011).

[24] C.Y. Wang, Quart. Appl. Math. 48, 601-610 (1990).

[25] M. Miklavčič and C.Y. Wang, Quart. Appl. Math. 64, 283-290 (2006).

[26] C.Y. Wang, Int. J. Non-Linear Mech. 43, 377- 382 (2008).

[27] A. Ishak, Y.Y Lok and I. Pop, Chem. Eng. Comm. 197, 1417-1427 (2010).

[28] T.R. Mahapatra, S.K. Nandy and A.S. Gupta, Meccanica 47, 1325-1335 (2012).

[29] S. Yao, T. Fang and Y. Zhong, Commun. Nonlinear Sci. Numer. Simulat. 16, 752-760 (2011).

[30] J. Zueco, O. Anwar Bég, H.S. Takhar and V.R. Prasad, Applied Thermal Engineering 29, 2808-2815 (2009).

[31] S. Pal, G. Balasubramanian and I.K. Puri, J. Chem. Phys. 136, 044901 (2012).

[32] H. Terada and T. Yamagata, Micromech. Microactuat.: Mech. Mach. Sci 2, 73-84 (2012).

[33] J.N. Reddy, An Introduction to the Finite Element Method, McGraw-Hill (2005).

[34] D. Gupta, L. Kumar, O Anwar Bég and B. Singh, Proc. IMechE. Part E: J Process Mechanical Engineering 228, 61-72(2014).

[35] O.A. Bég, T. A. Bég, R. Bhargava, S. Rawat and D. Tripathi, J. Mechanics in Medicine and Biology 12, 1-35 (2012).

[36] P. Rana and R. Bhargava, Applied Mechanics and Materials 110, 3679-3687 (2012).

[37] P. Rana, R. Bhargava and O.A. Bég, Proc. IMechE: Part N: J. Nanoengineering and Nanosystems 227,77-99 (2013).

[38] D. Yadav, G.S. Agrawal and R. Bhargava, Int. J. Engineering Science 49, 1171-1184 (2012).

[39] S. Kapoor, S. Rawat and S. Dhawan, Int. J. Computer App. 40, 1-14 (2012).

[40] K. Das, Comp. Math. App. 63, 255-267 (2012).

\begin{tabular}{llll} 
Nomenclature & & \\
$A$ & spin gradient viscosity & $T_{m}$ & temperature of the melting surface \\
$c_{f}$ & heat capacity of fluid & $T_{\infty}$ & temperature of the ambient fluid \\
$c_{p}$ & specific heat at constant pressure & $u$ & velocity in the $x$ - direction \\
$c_{s}$ & heat capacity of solid & $u_{e}$ & free stream velocity \\
$C$ & microinertia density & $u_{w}$ & velocity of stretching/shrinking sheet \\
$C_{f}$ & skin friction coefficient & $v$ & velocity in the $y$ - direction \\
$E c$ & Eckert number & $x$ & distance along the surface \\
$f$ & dimensionless velocity & $y$ & distance normal to the surface \\
$g$ & dimensionless microrotation & $\mathbf{G r e e k}$ symbols \\
$K$ & coupling constant parameter & $\gamma$ & spin gradient viscosity \\
$L$ & latent heat of the fluid & $\eta, \xi$ & similarity variables \\
$M$ & magnetic parameter & $\mu$ & the dynamic viscosity \\
$M e$ & melting parameter & $\rho$ & density of the fluid \\
& & $\kappa$ & thermal conductivity \\
\hline
\end{tabular}




\begin{tabular}{llll}
\hline$N$ & microrotation component & $v$ & kinematic viscosity \\
$N u_{x}$ & local Nusselt number & $\theta$ & dimensionless temperature \\
$\operatorname{Pr}$ & Prandtl number & $\phi$ & stream function \\
$q_{w}$ & heat flux & $\varepsilon$ & stretching/shrinking parameter \\
$\operatorname{Re}_{x}$ & local Reynolds number & $\tau_{w}$ & wall shear stress \\
$S$ & constant characteristic of the fluid & Subscripts \\
$T$ & temperature of the fluid & $\infty$ & surface condition \\
$T_{0}$ & temperature of the solid & & \\
\hline
\end{tabular}

